\title{
Volume of the ligamentum capitis femoris in osteoarthritic hip joints of adult dogs
}

\author{
J D Mande ${ }^{a^{*}}$, P M F Mbithi ${ }^{a}$, S W Mbugua ${ }^{a}$, I B J Buoro ${ }^{a}$ and P K Gathumbi ${ }^{b}$
}

\begin{abstract}
Ventrodorsal pelvic radiographs were made of 32 adult dogs under general anaesthesia. The hip joints were evaluated according to the severity of osteoarthritic changes graded as $0,1,2$ or 3 . The dogs were euthanased, the hip joints opened and the ligamentum capitis femoris dissected out in toto. The volume of each ligament was determined using a water displacement technique and the mean volume compared to the four radiographic grades of osteoarthritis. There was an inverse correlation $(r=-0.75)$ between the mean volume of the ligamentum capitis femoris and the increasing severity of osteoarthritis as assessed by radiography. The results confirmed the crucial role of radiography in the clinical evaluation of hip dysplasia and osteoarthritis in the adult dog. Assessment of the volume of the ligamentum capitis femoris revealed that it is an important tool for research in canine hip dysplasia and osteoarthritis.
\end{abstract}

Key words: hip dysplasia, hip joint, ligamentum capitis femoris, osteoarthritis, ventrodorsal pelvic radiography.

Mande J D, Mbithi P M F, Mbugua S W, Buoro I B J, Gathumbi P K Volume of the ligamentum capitis femoris in osteoarthritic hip joints of adult dogs. Journal of the South African Veterinary Association (2003) 74(1): 11-13 (En.). Department of Clinical Studies, Faculty of Veterinary Medicine, University of Nairobi, PO Box 29053, Nairobi, Kenya.

\section{INTRODUCTION}

Osteoarthritis is an important orthopaedic disease in dogs and human beings. The affected joint exhibits destruction of its articular cartilage, changes in the subchondral bone architecture, capsulitis and synovitis ${ }^{11,20}$. The disease occurs most frequently in the large, weight bearing joints of medium-sized to large dogs, but may affect any synovial joint. The best example of canine osteoarthritis is that occurring secondary to hip dysplasia ${ }^{2}$.

Joint laxity at a young age, trauma, heritability and genetic factors may modify the onset and progression of osteoarthritis and subsequent enzymatic degradation of the hyaline articular cartilage and synovium as the dog matures. Non-genetic factors, including body size, growth rate, nutrition, dietary anion gap, in-utero endocrine influences and muscle mass are also involved ${ }^{13,22}$. Despite these factors, the actual cause of canine hip dysplasia and osteoarthritis still remains unknown ${ }^{13}$. Body mass and joint laxity have been reported as signifi-

aDepartment of Clinical Studies, Faculty of Veterinary Medicine, University of Nairobi, PO Box 29053, Nairobi, Kenya.

'Department of Veterinary Pathology and Microbiology, Faculty of Veterinary Medicine, University of Nairobi, PO Box 29053, Nairobi, Kenya.

*Author for correspondence.

E-mail: jmande@wananchi.com

Received: September 2002. Accepted: January 2003. cant risk factors in 4 large-breed dogs, the risk of having osteoarthritis being 5 times greater in German shepherd dogs than in the other 3 breeds combined ${ }^{24}$. The probability of having osteoarthritis also increases with age. Mayhew et al. ${ }^{18}$ confirmed a contemporaneous association between a radiographic caudolateral curvilinear osteophyte on the femoral neck and osteoarthritis. Gustafson ${ }^{10}$ described the relationship between trauma and the pathogenesis of osteoarthritis. Mechanical damage to the chondrocytes and synoviocytes causes these cells to release enzymes and cytokines that contribute to the progressive degeneration of the hyaline articular cartilage, as well as the synovial membrane $e^{9,20}$

Clinical manifestations of canine hip dysplasia may either be asymptomatic, or acute in onset in young puppies. Chronically affected adult dogs may show a wobbly gait, hind-limb lameness, and atrophy of the thigh muscles and prominence of the femoral trochanters. In severe cases there may be alteration in the conformation of the pelvic limbs and reluctance to bear weight, and with progressive disability, the dogs may eventually become recumbent ${ }^{3}$.

The earliest recognisable change in the affected hip joints is joint laxity. This may be palpated (Ortolani sign, Barden's lift method) or visualised radiographically ${ }^{2}$. Subsequent radiological changes are those of degenerative joint disease. The order of subsequent changes commences with perichondral osteophyte formation, remodelling of the femoral head and neck, remodelling of the acetabulum and eventually sclerosis of the subchondral bone of the femoral head and acetabu$\operatorname{lum}^{2}$. Qualitative methods for assessing the severity of hip dysplasia include the subjective hip scores as established by the Orthopedic Foundation for Animals. Quantitative measures of passive hip joint laxity such as the Norberg angle and distractive index derived from newer stress-radiographic diagnostic methods are also often used ${ }^{4,22,23}$. More recent diagnostic techniques include a dorsolateral subluxation test using radiography ${ }^{7,8,16,17}$, dynamic ultra-sonography ${ }^{1}$, computed tomography ${ }^{8}$ and morphometric assessment of the canine hip joint using the dorsal acetabular rim view and the centre-edge angle ${ }^{19}$. A semiquantitative radiographic method of postoperative evaluation of traumatic hip dislocation was described in $\operatorname{dogs}^{6}$. Rasmussen et al. $^{21}$ used radiographic scoring criteria to evaluate the triple pelvic osteotomy procedure in canine patients with hip dysplasia. There is paucity of information on the use of similar criteria in adult dogs suffering from osteoarthritis of the hip joint.

The integrity and volume of the ligamentum capitis femoris has been reported in a colony of Labrador retriever dogs specifically kept for their predisposition to canine hip dysplasia ${ }^{14,15}$. However, there are no reports on the volume of the ligamentum capitis femoris in normal and osteoarthritic hip joints of adult dogs. The present study was designed to determine the relationship between the volume of the ligamentum capitis femoris and the radiographic features of normal and osteoarthritic hip joints in adult dogs in Kenya.

\section{MATERIALS AND METHODS}

Thirty-two adult German shepherd dogs that were either normal (8) or with hind-limb lameness (24) were used. Nine- 
teen were females and 13 males. The mean body mass was $27.1 \mathrm{~kg}$ while the mean age was 9.3 years. General anaesthesia was induced using intravenous injection of pentobarbitone sodium $\left(60 \mathrm{mg} / \mathrm{ml}, 25\right.$ mg per kg Sagatal ${ }^{\circledR}$, Rhône Meriux, Essex) and standard hip extended ventrodorsal radiography was performed. The severity of osteoarthritic changes within each hip joint was radiographically evaluated and assigned to grades $0,1,2$ or 3 , according to published criteria $^{21}$ (Appendix 1).

The dogs were euthanased using an intravenous injection of pentobarbitone sodium $\left(200 \mathrm{mg} / \mathrm{ml}\right.$, Euthatal ${ }^{\circledR}$ Rhône Meriux, Dublin). The animals that were clinically normal or with grade 1 osteoarthritis included in this part of the study had already been submitted for euthanasia for other medical reasons. The animals with severe osteoarthritis were euthanased due to poor prognosis and advanced age, making them no longer useful as guard dogs. The hip joints were dissected by cutting the muscles from around the pelvis and the femur. A band saw was used to cut the pubis, ilium and ischium to isolate the hip joint, while the femur was disarticulated at the stifle joint. The integrity of the ligamentum capitis femoris was determined by visual inspection. The ligament was dissected at its attachment to the acetabulum and at the fovea capitis of the femoral head. The volume of the ligament in millilitres was determined by measuring the volume of water displaced in a measuring cylinder as previously described ${ }^{5}$. The mean volume of each ligamentum capitis femoris was compared to each grade of osteoarthritis using analysis of variance. A correlation coefficient was used to determine the relationship between the mean volume of each ligamentum capitis femoris and the radiographic grade of osteoarthritis of its corresponding hip joint.

\section{RESULTS}

An intact ligamentum capitis femoris was present in $46(71.9 \%)$ and absent in 18 $(28.1 \%)$ of the hip joints examined in this study. Table 1 presents data on the volume of the ligamentum capitis femoris for each radiographic grade. The mean volume of the ligament in hip joints in radiographic grade 0 was higher $(0.821 \mathrm{~m} \ell)$ than that of grade $1(0.654 \mathrm{~m} \ell)$. Although 4 of 7 hip joints in radiographic grade 2 lacked ligaments, the mean volume of the ligamentum capitis femoris of hip joints in this group was $0.314 \mathrm{ml}$. The 13 hip joints in radiographic grade 3 lacked ligaments and the joints had moderate to severe osteoarthritis. The mean volume of the ligamentum capitis femoris

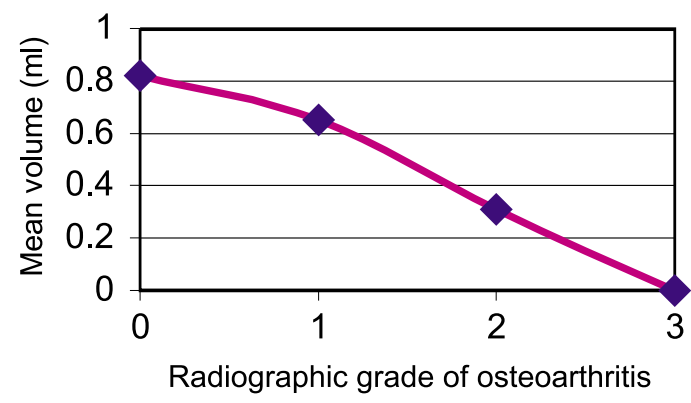

Fig. 1: Relationship between the mean volume $(\mathrm{ml})$ of ligamentum capitis femoris and radiographic grades of osteoarthritis of the hip joints in 32 adult German shepherd dogs.

differed highly significantly $(F=21.6, P<$ 0.0001 ) between groups. However, there was no significant difference between the mean volume of the ligamentum capitis femoris in those hip joints that were normal and those with only mild osteoarthritis.

The relationship between the mean volume of the ligamentum capitis femoris and the four radiographic grades is illustrated in Fig. 1. There is an inverse correlation $(r=-0.75)$ between the mean volume of the ligaments and the severity of osteoarthritis graded radiographically.

\section{DISCUSSION}

Canine hip dysplasia has been reported to be the most important cause of osteoarthritis in the hip joint of dogs in Europe and America ${ }^{22}$. This paper represents the 1st study of radiographic and pathological features of osteoarthritis of hip joints in adult dogs in Kenya.

Joint laxity has been reported to be an important etiological factor in the pathogenesis of canine hip dysplasia ${ }^{12}$. Although previous reports have provided data on ligamentum capitis femoris, young puppies were used that were specifically kept for their predisposition towards canine hip dysplasia. ${ }^{14,15}$ Ours is the 1st report describing the size of the ligamentum capitis femoris in normal and in osteoarthritic hip joints in adult German shepherd dogs.

The mean volume of the ligamentum

Table 1: Volume of the ligamentum capitis femoris $(\mathrm{m} \ell$ ) based on radiographic grades for osteoarthritis of the hip joints in 32 adult German shepherd dogs.

\begin{tabular}{cc}
\hline Radiographic grade & $\begin{array}{c}\text { Volume in me } \\
\text { mean } \pm \text { SD }(n), \text { range }\end{array}$ \\
\hline 0 & $0.821 \pm 0.3462(33)$ \\
& $0.4-1.5$ \\
1 & $0.654 \pm 0.2544(11)$ \\
& $0.4-1.0$ \\
2 & $0.314 \pm 0.5551(7)$ \\
& $0.2-1.5$ \\
3 & $(13)$ \\
& 0 \\
\hline
\end{tabular}

capitis femoris in normal hip joints (radiographic grade 0) was similar to that of hip joints with mild osteoarthritis in a previous study using young Labrador retriever dogs specifically kept for their genetic predisposition to canine hip dysplasia ${ }^{14,15}$. Although Lust et al. ${ }^{14}$ reported an increase in the mean volume of ligamentum capitis femoris in hip joints of young puppies with moderate to severe osteoarthritis, the results of our study demonstrated a decrease in the mean volume of the ligament in the hip joints of adult dogs with moderate and severe osteoarthritis. The ligament was small to absent and ruptured in the most severely affected hip joints. This relationship between the mean volume of ligamentum capitis femoris and the severity of radiographic features of osteoarthritis in the hip joints of adult dogs has not been reported previously in the veterinary literature.

This study provides additional information on how this important soft tissue structure is affected in hip joints with chronic osteoarthritis. It demonstrates the important role of assessing the volume of the ligament in research of canine hip dysplasia and osteoarthritis in adult dogs. Further, the results confirmed that ventrodorsal pelvic radiography is a valuable diagnostic tool for confirmation of the clinical signs of canine hip dysplasia and osteoarthritis. These data could be used for further epidemiological studies to determine the prevalence of the condition dogs and to evaluate the impact of current measures for evaluating canine hip dysplasia.

\section{ACKNOWLEDGEMENTS}

We thank the Department of Clinical Studies, University of Nairobi, for providing boarding facilities for the dogs and for radiography, and Mr Crispin Matere, Biometrics Unit, International Livestock Research Institute, Kenya, for statistical advice and analysis.

\section{REFERENCES}

1. Adams W M, Dueland R T, Daniels R, Fialkowski J P, Nordheim E V 2000 Comparison of two palpation, four radiographic and three ultrasound methods for early 
detection of mild to moderate canine hip dysplasia. Veterinary Radiology and Ultrasound 41: 484-490

2. Allan G 1998 Radiographic signs of joint disease. In Thrall D E (ed.) Textbook of veterinary diagnostic radiology. W B Saunders, Philadelphia: 169-188

3. Brinker W O, Piermattei D M, Flo G L 1990 Handbook of small animal orthopedics and fracture management. W B Saunders, Philadelphia: 355-375

4. British Veterinary Association/Kennel Club Hip Dysplasia Scheme: notes on procedure 1994 The Veterinary Journal 134: 389-391

5. Burton-Wurster N, Farese J P, Todhunter R J, Lust G 1999 Site specific variation in femoral head cartilage composition in dogs at high and low risk for development of osteoarthritis: insights into cartilage degeneration. Osteoarthritis and Cartilage 7: 486-497

6. Evers P, Kramek B A, Wallace L J, Johnston G R, King V 1997 Clinical and radiographic evaluation of intertrochanteric osteotomy in dogs: a retrospective study of 18 dogs. Journal of Veterinary Surgery 26: 217-222

7. Farese J P, Lust G, Williams A J, Dykes N L, Todhunter R J 1999 Comparison of measurements of dorsolateral subluxation of the femoral head and maximal passive laxity for evaluation of the coxofemoral joint in dogs. American Journal of Veterinary Research 60: 1571-1576

8. Farese J P, Todhunter R J, Lust G, Williams A J, Dykes N L 1998 Dorsolateral subluxation of hip joints in dogs measured in a weight-bearing position with radiography and computed tomography. Journal of Veterinary Surgery 27: 393-405

9. Fernandes J C, Martel-Pelletier J, Pelletier J P 2002 The role of cytokines in osteoarthritis pathophysiology. Biorheology 39: 237-246
10. Gustafson S 1993 Traumatic, septic and immune-mediated joint diseases. In Harari $\mathrm{J}$ (ed.) Surgical complications and wound healing in the small animal practice. W B Saunders, Philadelphia: 253-278.

11. Innes J F, Bacon D, Lynch C, Pollard A 2000 Long-term outcome of surgery from dogs with cranial cruciate ligament deficiency. The Veterinary Record 147: 325-328

12. Kealy R D, Lawler F, Ballam J M, Lust G, Smith G K, Biery D N, Olsson S E 1997 Fiveyear longitudinal study on limited food consumption and development of osteoarthritis in coxofemoral joints of dogs. Journal of the American Veterinary Medical Association 210: 222-225

13. Lust G 1997 An overview of the pathogenesis of canine hip dysplasia. Journal of the American Veterinary Medical Association 210: 1443-1445

14. Lust G, Beilman W T, Dueland D J, Farrell P W 1980 Intra-articular volume and hip joint instability in dogs with hip dysplasia. Journal of Bone and Joint Surgery 62-A: 576-582

15. Lust G, Summers B A 1981 Early, asymptomatic stage of degenerative joint disease in canine hip joints. American Journal of Veterinary Medicine 42: 1849-1855

16. Lust G, Todhunter R J, Erb H N, Dykes N L, Williams A J, Burton-Wurster N I, Farese J P 2001 Comparison of three radiographic methods for diagnosis of hip dysplasia in eight-month-old dogs. Journal of the American Veterinary Medical Association 219: 1242-1246

17. Lust G, Todhunter R J, Erb H N, Dykes N L, Williams A J, Burton-Wurster N I, Farese J P 2001 Repeatability of dorsolateral subluxation scores in dogs and correlation with macroscopic appearance of hip osteo- arthritis. American Journal of Veterinary Research 62: 1711-1715

18. Mayhew P D, McKelvie P J, Biery D N, Shofer F S, Smith G K 2002 Evaluation of a radiographic caudolateral curvilinear osteophyte on the femoral neck and its relationship to degenerative joint disease and distractive index in dogs. Journal of the American Veterinary Medical Association 220: 472-476

19. Meomartino L, Fatone G, Potena A, Brunetti A 2002 Morphometric assessment of the canine hip joint using the dorsal acetabular rim view and the centre-edge angle. Journal of Small Animal Practice 43: 2-6

20. Olee T, Hashimoto S, Quach J, Lotz M 1999 IL-18 is produced by articular chondrocytes and induces proinflammatory and catabolic responses. Journal of Immunology 162: 1096-1100

21. Rasmussen L M, Kramek B A, Lipowitz A J 1998 Preoperative variables affecting longterm outcome of triple pelvic osteotomy for treatment of naturally developing hip dysplasia in dogs. Journal of the American Veterinary Medical Association 213: 80-85

22. Smith G K 1997 Advances in diagnosing canine hip dysplasia. Journal of the American Veterinary Medical Association 210: 1451-1457

23. Smith G K, Hill C M, Gregor T P, Olsson K 1998 Reliability of the hip distraction index in two-month-old German shepherd dogs. Journal of the American Veterinary Medical Association 212: 1560-1563

24. Smith G K, Mayhew P D, Kapatkin A S, McKelvie P J, Shofer F S, Gregor T P 2001 Evaluation of risk factors for degenerative joint disease associated with hip dysplasia in German shepherd dogs, golden retrievers, Labrador retrievers and rottweilers. Journal of the American Veterinary Medical Association 219: 1719-1724

Appendix 1: Radiographic grading system for assessing the degree of coxofemoral osteoarthritis and canine hip dysplasia ${ }^{12}$.

\section{Radiographic grade Radiographic features}

Grade $0 \quad$ C-shaped acetabulum, dorsal rim rounded with a distinct femoral neck.

Grade 1 Shallow acetabulum or marked dorsal rim attenuation, moderately osteophytic acetabular margin, rounded femoral head, minimal osteophytes on the femoral neck.

Grade 2 Shallow acetabulum, or marked dorsal rim attenuation, moderately osteophytic acetabular margin, flattened femoral head, shortened femoral neck with osteophytes.

Grade 3

Flat acetabulum, severely osteophytic acetabular margin, marked flattened or irregular femoral head, severely shortened femoral neck with osteophytes. 